THE FLETCHER JONES FOUNDATION

HUMAN ITIES | MPR INT

The Fletcher Jones Foundation has endowed this imprint to foster innovative and enduring scholarship in the humanities. 
THE PUBLISHER AND THE UNIVERSITY OF CALIFORNIA PRESS

FOUNDATION GRATEFULLY ACKNOWLEDGE THE GENEROUS

SUPPORT OF THE FLETCHER JONES FOUNDATION IMPRINT

IN HUMANITIES. 
UNDERSTANDING RELIGION 



\section{UNDERSTANDING RELIGION}

Theories and Methods for Studying Religiously Diverse Societies

Paul Hedges

प UNIVERSITY OF CALIFORNIA PRESS 
University of California Press

Oakland, California

(C) 202I by Paul Hedges

Library of Congress Cataloging-in-Publication Data

Names: Hedges, Paul (Paul Michael), I970- author.

Title: Understanding religion : theories and methods for studying religiously diverse societies / Paul Hedges.

Description: Oakland, California : University of California Press, [202I] | Includes bibliographical references and index.

Identifiers: LCCN 2020032939 (print) | LCCN 2020032940 (ebook) |

ISBN 9780520298897 (hardcover) | ISBN 97805202989 Io (paperback) | ISBN 978052097086I (ebook)

Subjects: LCSH: Religious pluralism—Case studies. | Religions-

Relations-Case studies. | Religion and sociology—Case studies.

Classification: LCC BL4IO .H46 202I (print) | LCC BL4IO (ebook) |

DDC $200.7 \mathrm{I}$ - dc23

LC record available at https://lccn.loc.gov/2020032939

LC ebook record available at https://lccn.loc.gov/2020032940

Manufactured in the United States of America

$\begin{array}{lllllllll}29 & 28 & 27 & 26 & 25 & 24 & 23 & 22 & 21\end{array}$

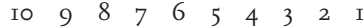

